\title{
RADIATIVE TRANSFER IN NOVA ENVELOPES
}

\author{
R.P. Harkness \\ Department of Astrophysics, Oxford University, South \\ Parks Road, Oxford, OXI 3RQ UK.
}

\section{INTRODUCTION}

This paper presents some preliminary results of work aimed at producing model atmospheres for the steady-state, constant luminosity continuous ejection model of classical novae. The results are generally. applicable to any object with an outflowing atmosphere driven predominantly by radiation pressure in an optically thick continuum. In particular it is aimed at removing the limitations of assuming blackbody photospheric boundary conditions and strict LTE and the problems associated with their definition.

The basic assumptions follow from the model of classical novae suggested by Bath and Shaviv (1976), namely, that all classical novae are semi-detached binary systems containing a compact white dwarf and a Roche-lobe-filling red star with a separation of $10^{11} \mathrm{~cm}$ and that following the initial eruption the classical nova envelope can be described in terms of a continuously ejected outflowing wind, which at maximum light is optically thick to a radius much greater than that of the underlying binary system. The correlation between the appearance of characteristic emission lines and the optical decline stage below maximum (Payne-Gaposhkin 1957; McLaughlin 1960) is then ascribed to the overall re-distribution of flux further into the IV as the rate of mass loss $(\dot{m})$ declines. This causes the photospheric radius to contract. While the bolometric luminosity remains constant and of the order of the Eddington luminosity. The assumption of constant total luminosity is supported by UV observations of Nova Serpentis 1970 (Gallagher and Code 1974) and those of Nova Cygni 1975 (Wu and Kester 1977).

\section{FREQUENCY-INDEPENDENT MODELS}

In a series of papers (Bath and Shaviv 1976, Bath 1978, Ruggles and Bath 1979) the basic model has been developed from order of magnitude calculations in support of the idea, to detailed calculations on the structure of optically thick winds. The overall picture is generally supported by the results of hydrodynamic models involving nuclear reaction networks (Prialnik et al.1978, Starrfield et al.1974, 1976, 1978) which can lead to continuing mass loss (particularly in the case of alow novae) though these models all suffer from difficulties at the outer boundary.

Since the results presented here rely to some extent on the structure of optically thick winds as derived by Ruggles and Bath 
(1979) a short review of the assumptions of their work is given below, paying particular attention to those which have importance with regard to radiative transfer:

(1) It is assumed that the nova outburst can be described in terms of a quasi-stationary sequence of spherically symetric, steady-state winds.

(2) The "photosphere" is a sharply defined boundary, below which strict LTE obtains and above which photons escape freely to infinity. See section IV for comments on the validity of this assumption.

(3) Energy transport is by radiative diffusion ( $J=3 K)$.

(4) The velocity of the material remains constant beyond the "photosphere", $v=v_{s}$.

(5) Only the continuum is considered; frequency integrated opacities and frequency integrated equation of transfer are used. (Castor 1972).

These assumptions lead to two surface boundary conditions:

(i) a "photospheric" boundary condition

$$
r_{3}=\frac{\bar{\kappa} \dot{m}}{8 \pi v_{s}}
$$

(ii) a blackbody boundary condition

$$
L_{8}=\operatorname{Tacr}_{8}^{2} T_{8}^{4}
$$

Solutions to the resulting system of equation are required to obey both these boundary conditions simultineously.

This approach has the great advantage that the equation of transfer can be solved in spherical geometry including all terms to order $\mathrm{v} / \mathrm{c}$, although in frequency-integrated form. The disadvantages are that it is restricted to LTE and radiative diffusion, yields no spectral information and cannot include a full treatment of scattering. However, the solutions can be expected to be increasingly dependable at increasing optical depths, where most of the acceleration takes place. In fact these models show that the radius of the critical point in the flow must be close to the surface of the white dwarf component in order to achieve the observed terminal velocities from a trans-sonic flow and also demonstrate the importance of including the advection terms in the equation of transfer at large optical depths. In all but the Lowest $\dot{m}$ models (i.e. those winds with the least optical depth at the critical point) it is found that $v \sim$ constant well below the "photosphere" and that in this region the flow is essentially adiabatic. 
To investigate frequency-dependent effects, departures from blackbody emission, non-LTE etc., over the entire range of optical depth while maintaining computational feasibility, in the work which follows the density as a function of radius is taken from the models of Ruggles and Bath. Also, the aberration, advection and frequencyderivative terms are omitted from the equation of transfer, reducing it to the static equation of transfer in spheical geometry (3), thereby greatly simplifying its solution.

$$
\mu \frac{\partial I_{\nu}}{\partial r}+\frac{\left(1-\mu^{2}\right)}{r} \frac{\partial I_{\nu}}{\partial \mu}=\eta_{\nu}-X_{\nu} I_{\nu}
$$

In these preliminary models it is further assumed that the flow is adiabatic in the $v=$ conscant region.

The equation of transfer is solved using the variable Eddington factor method. A formal solution of the equation is carried out, using a given source function, along impact parameters tangent to spherical shelis on which the physical variables are defined

(Mihalas 1978). The sphericity factor due to Auer (1971) can then be calculated and the moment equations solved including any scattering terms. The source function can then be re-evaluated and a new formal solution carried out. The process is iterated to convergence.

A number of numerical difficulties are presented by the very large extension factors which have to be considered in these models. The radius of the surface she 11 may typically be 103-104 times larger than the inner "core" radius. In most previous work on extended spherical atmospheres this factor is typically $\sim 10^{2}$.

\section{EMERGENT FLUX RESULTS AND DISCUSSION}

A typical emergent flux for a high $\dot{m}$ wind from a $0.41 \mathrm{M}_{0}$ white dwarf is shown in Fig.1. Only LTE results are available at present. A number of features are immediately apparent. Firstly, the spectra displays emission edges (Cassinelli 1971, Cassine11i and Hartmann 1975). These are due to geometrically-induced emission (Gebbie and Thomas 1968). The effect can be very large in these atmospheres and as a result it is not possible to attach too much significance to a "photosphere" defined at, say, a Rosseland mean optical depth $\tau_{\mathrm{R}} \mathbf{l}$. This presents a problem for the type of solution outlined in section II. The frequency-dependent result has no such problem, since the outer boundary condition used in solving the transfer equation states only that the re is no incoming radiation.

The second feature is that the spectrum is characteristically "flat" (Cassinalli 1971), with large absorption edges in the UV, due to neutral metals in this case. Early NLTE results show that, at least for hydrogen ground states are extremely underpopulated, so the inclusion of the full NLTE is expected to drastically alter the appearance of the emergent flux in the UV. 


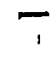

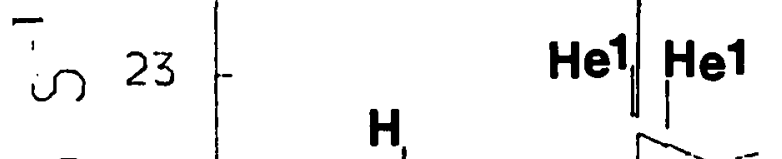

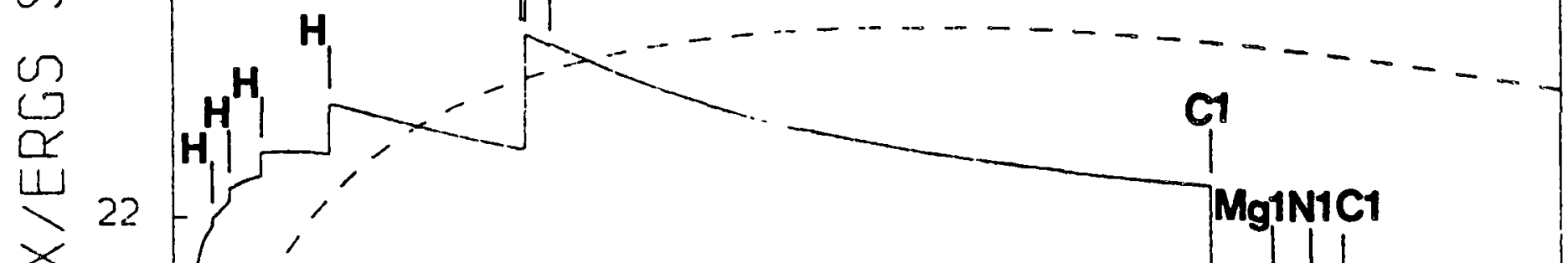

$\underline{x}$

21
20

19

18

$M_{H D}=0.4100 E 00$

$R_{W D}=0.1075 E 10$

$17-\dot{M}^{W}=0.3162 E 22$

$R_{C}=0.1075 E 10$
$R_{2 / 3}=0.2966 E ! 2$
$T_{2 / 3}=0.3095 E 05$

16

0.0

Fig 1

FREQUENCY/HZ $\left(* 10^{15}\right)$ 
Sequences of models with decreasing $\dot{m}$ exhibit the same gross features, the spectra becoming increasingly "flat" with an increasing proportion of the flux radiated at wavelengths less than $3000 \AA$. Ali models produced so far have near-solar abundances. The results of Prialnik et al. and Starrfield et al. indicate that various CNO abundances will be significantly affected by an outburst and choosing suitable abundances remains a problem. A further difficulty is the absence of line blanketing which will have particular importance in the UV. Also, in the determination of the structure of the wind, line opacities and their contribution to the radiation force were, of course, neglected since only a frequency-integrated treatment of the dynamics is possible with the approach described here.

\section{REFERENCES}

Auer, L.H. 1971, J. Quant.Spectrosc. Rad. Tranisf., 11, 573. Bath, G.T. 1978, Monthly Notices Roy. Astron. Soc., 182, 35.

Bath, G.T., Shaviv, G. 1976, Monthly Notices Roy. Astron. Soc., 175, 305.

Cassinelli, J.P. 1971, Astrophys. J. 165, 265.

Cassinelli, J.P., Hartmann, L. 1975, Astrophys. J. 202, 718.

Castor, J.I. 1972, Astrophys. J. 178, 779.

Gallagher, J.S., Code, A.D. 1974, Astrophys. J. 189, 303.

Gebbie, K.B., Thomas, R.N. 1968, Astrophys. J. 164, 285.

Mclaughlin, D.B. 1960, Stars and Stellar Systems VI.

Michalas, D. 1978, Stellar Atmospheres (2nd Edition) Freeman.

Payne-Gaposchk1n, C. 1957, The Galactic Noyae, North Holland, Amsterdam.

Prialnik, D., Shara, M.N., Shaviv, G. 1978, Astron. Astrophys. 62, 339.

Ruggles, C.L.N., Bath, G.T. 1979, Astron. and Astrophys. in press.

Starrfield, S.G., Sparks, W.M., Truran, J.W. 1974 Astrophys. J. Supp1., 261

. 1976 IAU Symp. No. 73.

Structure and Evolution of Close Binary Systems. eds. P. Eggleton, S. Mitton, J.A.J. Whelan. D. Reidel, Dordrecht, Holland.

- 1978 Astrophys. J. 220, 1065.

Wu, C.C. Kester, D. 1977, Astron. Astrophys., 58, 331. 